\title{
An unusual complication of anesthesia: Unilateral spinal myoclonus
}

\section{Bahadır KÖSEM, Hatice KILINÇ}

To the Editor,

Myoclonus is comprised of sudden, involuntary contractions of a group of muscles, a single muscle, or part of a muscle. Spinal, focal, or segmental myoclonus has specific features that distinguish it from other forms of more generalized myoclonus. It is often restricted to one somatic region due to the pathology at the involved level of the spinal cord. The possible causes are tumor, infection, trauma and degenerative processes. ${ }^{[1]}$ Drugs administered through intrathecal and epidural routes, neuraxial high dose opioid therapy, indwelling spinal or epidural catheters can occasionally cause myoclonus. ${ }^{[2,3]}$ Herein we presented a patient with unilateral spinal myoclonus due to spinal anesthesia.

A 36-year-old woman underwent surgery for left leg varices. She had no previous surgeries, no significant medical history, no allergies and no previous neurological disease. She was premedicated with 3 mg midazolam following blood pressure, $\mathrm{SpO}_{2}$ and ECG monitoring. She then received $3 \mathrm{ml}$ of $0.5 \%$ hyperbaric bupivacaine given by a $25 \mathrm{G}$ spinal needle through the L4-L5 interval on the left side and left fowler position. No opioids were added. A sensory block up to the thoracic 6 level (pin prick technique) associated with motor block was obtained prior to the start of surgery. The surgery proceeded uneventful, as well as the intraoperative course. One hour after surgery, the patient was presented with sudden, involuntary and unilateral rhytmic contractions of right leg. This movements were not associated with voluntary movements or reflex stimuli, and there was no evidence of weakness, impairment of vibration, pinprick of touch sense, or cerebellar or cranial nevre dysfunction. Conventional brain and spinal cord magnetic resonance imaging (MRI) disclosed no abnormalites. In addition laboratory tests including serum B12, calcium, magnesium and potasium levels were both normal. According to laboratory and clinical investigation spinal myoclonus due to spinal anesthesia was diagnosed. Five hours after surgery, voluntary movement of right leg associated with myoclonus was disappeared. During the course of spinal myoclonus no medication was used.

Acute spinal myoclonus following regional anaesthesia is extremely rare, and treatment should be directed at the aetiology. Anaesthetists should watch out for this anaesthetic complication, especially in patients with underlying vitamin deficiency or neuromuscular disease. Anaesthetists who are unfamiliar with this rare complication should be reassured that it may be treated successfully with midazolam. ${ }^{[4]}$ In addition in the literature some authors suggested that patient with spinal myoclonus can be followed up without any treatment. ${ }^{[5]}$ Unilateral spinal myoclonus following regional anaesthesia is extremely rare. In conclusion the clinicians should be kept in mind this unusual complication especially in patient with regional anesthesia. 


\section{References}

1. Hoehn MM, Cherington M. Spinal myoclonus. Neurology 1977;27(10):942-6.

2. Alfa JA, Bamgbade OA. Acute myoclonus following spinal anaesthesia. Eur J Anaesthesiol 2008;25(3):256-7.

3. Ford B, Pullman SL, Khandji A, Goodman R. Spinal my- oclonus induced by an intrathecal catheter. Mov Disord 1997;12(6):1042-5.

4. Caviness JN, Brown P. Myoclonus: current concepts and recent advances. Lancet Neurol 2004;3(10):598-607.

5. CelikY, Bekir Demirel C, Karaca S, Kose Y. Transient segmental spinal myoclonus due to spinal anaesthesia with bupivacaine. J Postgrad Med 2003;49(3):286. 